\title{
On the well-posedness of the Holm-Staley $b$-family of equations
}

Hasan Inci

To cite this article: Hasan Inci (2016) On the well-posedness of the Holm-Staley $b$-family of equations, Journal of Nonlinear Mathematical Physics 23:2, 213-233, DOI: https://doi.org/10.1080/14029251.2016.1161261

To link to this article: https://doi.org/10.1080/14029251.2016.1161261

Published online: 04 January 2021 


\title{
On the well-posedness of the Holm-Staley $b$-family of equations
}

\author{
Hasan Inci \\ EPFL SB MATHAA PDE \\ MA C1 627 (Bâtiment MA) \\ Station 8 \\ CH-1015 Lausanne \\ Switzerland \\ hasan.inci@epfl.ch
}

Received 4 January 2016

Accepted 26 January 2016

\begin{abstract}
In this paper we consider the Holm-Staley $b$-family of equations in the Sobolev spaces $H^{s}(\mathbb{R})$ for $s>3 / 2$. Using a geometric approach we show that, for any value of the parameter $b$, the corresponding solution map, $u(0) \mapsto$ $u(T)$, is nowhere locally uniformly continuous.
\end{abstract}

Keywords: diffeomorphism group, solution map

2000 Mathematics Subject Classification: 35Q35

\section{Introduction}

Holm and Staley introduced in [22] the following family of equations

$$
u_{t}-u_{x x t}+(b+1) u u_{x}=b u_{x} u_{x x}+u u_{x x x}
$$

or rewritten

$$
u_{t}+u u_{x}=\left(1-\partial_{x}^{2}\right)^{-1}\left(-b u u_{x}+(b-3) u_{x} u_{x x}\right)
$$

related to shallow water, where

$$
u(t, x) \in \mathbb{R}, \quad(t, x) \in \mathbb{R}^{2}
$$

denotes the velocity field and $b \in \mathbb{R}$ is a parameter. For $b=2$ we get the Camassa-Holm equation (see e.g. [5]) and for $b=3$ the Degasperis-Procesi equation (see e.g. [14]). Both equations arise as integrable model equations for the propagation of shallow water waves of moderate amplitude. For the physical aspects see [9] and for the integrability aspects see [10,11]. In his seminal paper [3], Arnold showed that the Euler equation can be interpreted as an equation for a flow on groups of diffeomorphisms of the underlying space. It turns out that quite a few nonlinear evolution equations such as the KdV (see [30]), the Camassa-Holm equation (see [12, 28]) or various other equations of mathematical physics (see $[16,18,19]$ ) can be treated by such a geometric approach. Recently, in [17], it has been shown that this is also the case for the Holm-Staley $b$-family. In [7], Constantin used such a geometric approach to get local well-posedness and blow-up results for the CamassaHolm equation on $\mathbb{R}$. In this paper we will use these methods to prove our results. 
The Cauchy problem for (1.1) in $H^{s}(\mathbb{R}), s>3 / 2$, with initial value $u_{0} \in H^{s}(\mathbb{R})$, is to find $u \in$ $C^{0}\left([0, T], H^{s}(\mathbb{R})\right)$ for some $T>0$, such that we have the following identity in $H^{s-1}(\mathbb{R})$

$$
u(t)=u_{0}+\int_{0}^{t}\left(1-\partial_{x}^{2}\right)^{-1}\left(-b u u_{x}+(b-3) u u_{x x}\right)-u u_{x} d s
$$

for all $t \in[0, T]$. Here we regard $u_{x} u_{x x}$ as an element of $H^{s-2}(\mathbb{R})$ even if $3 / 2<s<2-$ see Appendix A. With this in mind and the fact that $H^{s-1}(\mathbb{R})$ is a Banachalgebra we see that the integrand in (1.3) is an element of $C^{0}\left([0, T], H^{s-1}(\mathbb{R})\right)$. Hence (1.3) makes sense and we have actually $u \in C^{1}\left([0, T], H^{s-1}(\mathbb{R})\right)$.

Concerning the well-posedness of (1.1) we have the following result - see also [29]

Theorem 1.1. Let $s>3 / 2$. For any given $u_{0} \in H^{s}(\mathbb{R})$ there is $a T>0$ and a unique solution $u \in C^{0}\left([0, T], H^{s}(\mathbb{R})\right.$ to the Cauchy problem $(1.1)$ with initial value $u(0)=u_{0}$. The $T$ can be chosen to be the same in a neighborhood $U \subseteq H^{s}(\mathbb{R})$ of $u_{0}$. Moreover the map

$$
U \rightarrow C^{0}\left([0, T], H^{s}(\mathbb{R})\right), \quad u_{0} \mapsto u
$$

is continuous.

Now one asks whether the map mentionned in the above theorem is more than continuous, e.g. $C^{1}$ or at least locally lipschitz. We have the following negative answer.

Theorem 1.2. Denote by $U_{T} \subseteq H^{s}(\mathbb{R})$ the set of initial values for which we have existence up to at least $T$. Then the map $U_{T} \rightarrow H^{s}(\mathbb{R}), u_{0} \mapsto u(T)$, mapping the initial value to the time $T$ value of the solution, is nowhere locally uniformly continuous.

Results saying that the solution map $u_{0} \mapsto u(T)$ has not the property to be uniformly continuous on bounded sets is known. On the circle this was proved in the case $b=2$ (Camassa-Holm equation) in [20] and in the case $b=3$ (Degasperis-Procesi equation) in [13] for $s \geq 2$. For the $b$-family on the line this was proved in [32].

\section{The geometric framework}

In [23] we considered for $s \in \mathbb{R}, s>3 / 2$, the space $\mathscr{D}^{s}(\mathbb{R})$ (cf M. Cantor [6]) given by

$$
\begin{aligned}
\mathscr{D}^{s}(\mathbb{R}) & :=\left\{\varphi: \mathbb{R} \rightarrow \mathbb{R} C^{1}-\text { diffeomorphism } \mid \varphi_{x}>0 \text { and } \varphi(x)-x \in H^{s}(\mathbb{R})\right\} \\
& =\left\{\varphi(x)=x+f(x) \mid f \in H^{s}(\mathbb{R}) \text { and } \varphi_{x}>0\right\}
\end{aligned}
$$

where $H^{s}(\mathbb{R})$ denotes the space of Sobolev functions on $\mathbb{R}$ of class $s$. In terms of the Fourier transform this reads as (see e.g. [2])

$$
H^{s}(\mathbb{R})=\left\{f \in L^{2}(\mathbb{R}) \mid\left(1+\xi^{2}\right)^{s / 2} \hat{f}(\xi) \in L^{2}(\mathbb{R} ; \mathbb{C})\right\}
$$

where $\hat{f}$ is the Fourier transform of $f$. Equipped with the scalar product (taking the real part)

$$
\langle f, g\rangle_{s}=\Re \int_{\mathbb{R}}\left(1+\xi^{2}\right)^{s} \hat{f}(\xi) \overline{\hat{g}(\xi)} d \xi
$$


it becomes a Hilbert space. Then

$$
\mathscr{D}^{s}(\mathbb{R})-i d=\left\{\varphi(x)-x \mid \varphi \in \mathscr{D}^{s}(\mathbb{R})\right\} \subseteq H^{s}(\mathbb{R})
$$

is open and thus has naturally the structure of a analytic Hilbert manifold (cf e.g. [23]). Moreover $\mathscr{D}^{s}(\mathbb{R})$ is a topological group under composition. More precisely, for any $k \in \mathbb{Z}_{\geq 0}$,

$$
H^{s+k}(\mathbb{R}) \times \mathscr{D}^{s}(\mathbb{R}) \rightarrow H^{s}(\mathbb{R}),(f, \varphi) \mapsto f \circ \varphi
$$

is a $C^{k}$-map. In the literature the partial maps of the compostion map are referred to as the $\alpha$-resp. the $\omega$-lemma - see [15].

In the following we need the notion of sprays. These are special vectorfields on the tangent bundle - see e.g. [27]. In our case we have the following identification for the tangent bundle of $\mathscr{D}^{s}(\mathbb{R})$

$$
T \mathscr{D}^{s}(\mathbb{R})=\mathscr{D}^{s}(\mathbb{R}) \times H^{s}(\mathbb{R}) .
$$

Thus a spray can be defined by a map $S$ with the following structure

$$
\begin{aligned}
S: \mathscr{D}^{s}(\mathbb{R}) \times H^{s}(\mathbb{R}) & \rightarrow H^{s}(\mathbb{R}) \times H^{s}(\mathbb{R}) \\
(\varphi, v) & \mapsto\left(v, \Gamma_{\varphi}(v, v)\right)
\end{aligned}
$$

where $\Gamma$, called the Christoffel map of the spray $S$, is a map

$$
\begin{aligned}
\Gamma: \mathscr{D}^{S}(\mathbb{R}) & \rightarrow L\left(H^{s}(\mathbb{R}), H^{s}(\mathbb{R}) ; H^{s}(\mathbb{R})\right) \\
\varphi & \mapsto \Gamma_{\varphi}(\cdot, \cdot)
\end{aligned}
$$

with values in the continuous $H^{s}(\mathbb{R})$-valued bilinear forms on $H^{s}(\mathbb{R})$. Since we are just interested in the quadratic form $\Gamma_{\varphi}(v, v)$ we assume $\Gamma_{\varphi}$ to be symmetric. The integral curves of $S$ projected on $\mathscr{D}^{s}(\mathbb{R})$ are called the geodesics of $S$. Like in the case of a Riemannian manifold we have also here the notion of an exponential map - see e.g. [27]. More precisely, the equation of the geodesics reads as

$$
\varphi_{t t}=\Gamma_{\varphi}\left(\varphi_{t}, \varphi_{t}\right)
$$

where the subscript $t$ denotes differentiation with respect to $t$. For analytic $S$ the Picard iteration gives local solutions of (2.1) with initial data $\varphi(0)=i d \in \mathscr{D}^{s}(\mathbb{R})$ and $\varphi_{t}(0)=v \in H^{s}(\mathbb{R})$. Because of the scaling properties of (2.1) there exists a neighborhood $V$ of $0 \in H^{s}(\mathbb{R})$ such that the initial value problem

$$
\left\{\begin{array}{c}
\varphi_{t t}=\Gamma_{\varphi}\left(\varphi_{t}, \varphi_{t}\right) \\
\varphi(0)=i d, \varphi_{t}(0)=v
\end{array}\right.
$$

admits a solution on the time interval $[0,1]$ for all $v \in V$. This allows us to define the exponential map exp as

$$
\begin{aligned}
\exp : V & \rightarrow \mathscr{D}^{s}(\mathbb{R}) \\
v & \mapsto \varphi_{v}(1)
\end{aligned}
$$

where $\varphi_{v}$ is the solution of (2.2). Because of the analytic dependence of solutions of (2.2) on the initial values, see [27], we know that exp is a smooth map. Moreover the derivative of exp at $0 \in$ 
$H^{s}(\mathbb{R})$ is the identity, i.e.

$$
d_{0} \exp : H^{s}(\mathbb{R}) \rightarrow H^{s}(\mathbb{R}), \quad v \mapsto v
$$

where we have identified $T_{i d} \mathscr{D}^{s}(\mathbb{R})$ with $H^{s}(\mathbb{R})$. By the inverse function theorem for Banach spaces, see [27]), exp is an analytic diffeomorphism between a neighborhood $U$ of $0 \in H^{s}(\mathbb{R})$ and a neighborhood $V$ of $i d \in \mathscr{D}^{s}(\mathbb{R})$, i.e.

$$
\exp : U \rightarrow V
$$

is an analytic diffeomorphism.

For our purpose we define $\Gamma$ at $i d \in \mathscr{D}^{s}(\mathbb{R})$ for $v \in H^{s}(\mathbb{R})$ with $s>3 / 2$ by

$$
\Gamma_{i d}(v, v)=\left(1-\partial_{x}^{2}\right)^{-1}\left(-b v v_{x}+(b-3) v_{x} v_{x x}\right)
$$

which is a continuous $H^{s}(\mathbb{R})$-valued quadratic form on $H^{s}(\mathbb{R})$. For $\varphi \in \mathscr{D}^{s}(\mathbb{R})$ arbitrary, $\Gamma_{\varphi}$ is defined by

$$
\Gamma_{\varphi}(v, v)=\left(\Gamma_{i d}\left(v \circ \varphi^{-1}, v \circ \varphi^{-1}\right)\right) \circ \varphi .
$$

In view of the poor regularity properties of the composition map, apriori it is not clear if $\Gamma$ defines a smooth spray. In the next section we verify that this is indeed the case. In the following we will make some formal computations to show how the geodesics of $S$ and solutions to (1.1) are related. Assume that $\varphi:[0, T] \rightarrow \mathscr{D}^{s}(\mathbb{R})$ solves the initial value problem (2.2). Then we have for $u:=\varphi_{t} \circ \varphi^{-1}$

$$
\begin{aligned}
\varphi_{t t} & =(u \circ \varphi)_{t}=u_{t} \circ \varphi+u_{x} \circ \varphi \cdot \varphi_{t} \\
& =u_{t} \circ \varphi+u_{x} \circ \varphi \cdot u \circ \varphi .
\end{aligned}
$$

Substituting this expression into equation (2.1) we get

$$
\begin{aligned}
u_{t} \circ \varphi+u_{x} \circ \varphi \cdot u \circ \varphi & =\Gamma_{\varphi}\left(\varphi_{t}, \varphi_{t}\right) \\
& =\Gamma_{i d}\left(\varphi_{t} \circ \varphi^{-1}, \varphi_{t} \circ \varphi^{-1}\right) \circ \varphi
\end{aligned}
$$

or by (2.3) equivalently

$$
u_{t}+u u_{x}=\left(1-\partial_{x}^{2}\right)^{-1}\left(-b u u_{x}+(b-3) u_{x} u_{x x}\right)
$$

which is equation (1.2). In the next section we show that by this approach one gets local wellposedness results for equation (1.1).

\section{Local wellposedness of the $b$-family of equations}

In this section we establish local existence and uniqueness for the Cauchy problem

$$
u_{t}+u u_{x}=\left(1-\partial_{x}^{2}\right)^{-1}\left(-b u u_{x}+(b-3) u u_{x x}\right), \quad u(0)=u_{0} \in H^{s}(\mathbb{R})
$$

in $H^{s}(\mathbb{R}), s>3 / 2$. 
Theorem 3.1. The spray S given by

$$
S: \mathscr{D}^{s}(\mathbb{R}) \times H^{s}(\mathbb{R}) \rightarrow H^{s}(\mathbb{R}) \times H^{s}(\mathbb{R}), \quad(\varphi, u) \mapsto\left(v, \Gamma_{\varphi}(u, u)\right)
$$

where

$$
\Gamma_{\varphi}(u, u)=R_{\varphi}\left(1-\partial_{x}^{2}\right)^{-1}\left(-b\left(R_{\varphi^{-1}} u\right) \cdot\left(R_{\varphi^{-1}} u\right)_{x}+(b-3)\left(R_{\varphi^{-1}} u\right)_{x} \cdot\left(R_{\varphi^{-1}} u\right)_{x x}\right)
$$

is analytic.

Recall that we use the notation $R_{\varphi}$ for right translation $H^{s}(\mathbb{R}) \rightarrow H^{s}(\mathbb{R}), f \mapsto f \circ \varphi$. Before proving Theorem 3.1 we show the following lemma.

Lemma 3.1. Let $s>3 / 2$. For $k=1,2$ the map

$$
\delta^{(k)}: \mathscr{D}^{s}(\mathbb{R}) \times H^{s}(\mathbb{R}) \rightarrow H^{s-k}(\mathbb{R}), \quad(\varphi, f) \mapsto R_{\varphi} \partial_{x}^{k} R_{\varphi^{-1}} f
$$

is analytic.

Proof. Consider first the case $k=1$. Then we have

$$
\delta^{(1)}(\varphi, f)=R_{\varphi} \partial_{x} R_{\varphi^{-1}} f=\frac{f_{x}}{\varphi_{x}}
$$

and this is an analytic expression in $\varphi$ and $f$. Similarly for $k=2$ we have we have

$$
\delta^{(2)}(\varphi, f)=R_{\varphi} \partial_{x}^{2} R_{\varphi^{-1}} f=\frac{f_{x x}}{\varphi_{x}}-\frac{f_{x} \cdot \varphi_{x x}}{\left(\varphi_{x}\right)^{3}}
$$

which also holds in the case $s<2-$ see Appendix A for the conventions in this case. We see from the expressions for $\delta^{(2)}(\varphi, f)$ that it is indeed analytic.

Now we can give the proof Theorem 3.1.

Proof of Theorem 3.1. Consider the continuous symmetric bilinear map

$$
\begin{aligned}
B: H^{s}(\mathbb{R}) \times H^{s}(\mathbb{R}) & \rightarrow H^{s-2}(\mathbb{R}) \\
(u, v) & \mapsto \frac{1}{2}\left(-b u v_{x}-b v u_{x}\right)+\frac{1}{2}\left((b-3) u v_{x x}+(b-3) v u_{x x}\right) .
\end{aligned}
$$

That the range of this expression is in $H^{s-2}(\mathbb{R})$ and its continuity follow from the Banach algebra properties of $H^{s}(\mathbb{R})$ - see e.g. [2] (for $s<2$ see Appendix A). From Lemma 3.1 we know that the 
map

$$
\begin{aligned}
\mathscr{D}^{s}(\mathbb{R}) & \rightarrow L\left(H^{s}(\mathbb{R}), H^{s}(\mathbb{R}) ; H^{s-2}(\mathbb{R})\right) \\
\varphi & \mapsto\left[(u, v) \mapsto R_{\varphi} B\left(R_{\varphi^{-1}} u, R_{\varphi^{-1}} v\right)\right]
\end{aligned}
$$

is analytic. Again using Lemma 3.1 we get that

$$
\begin{aligned}
\mathscr{D}^{s}(\mathbb{R}) & \rightarrow L\left(H^{s}(\mathbb{R}) ; H^{s-2}(\mathbb{R})\right) \\
\varphi & \mapsto\left[u \mapsto R_{\varphi}\left(1-\partial_{x}^{2}\right) R_{\varphi^{-1}} u\right]
\end{aligned}
$$

is analytic. Hence

$$
\begin{aligned}
\mathscr{D}^{s}(\mathbb{R}) & \rightarrow L\left(H^{s}(\mathbb{R}), H^{s-2}(\mathbb{R})\right) \\
\varphi & \mapsto\left[u \mapsto R_{\varphi}\left(1-\partial_{x}^{2}\right) R_{\varphi^{-1}} u\right]
\end{aligned}
$$

is analytic. Note that $u \mapsto R_{\varphi}\left(1-\partial_{x}^{2}\right) R_{\varphi^{-1}} u$ is invertible with inverse given by $v \mapsto R_{\varphi}(1-$ $\left.\partial_{x}^{2}\right)^{-1} R_{\varphi^{-1}} v$. Now as inversion of linear operators is an analyitc process we see that the map

$$
\begin{aligned}
\mathscr{D}^{s}(\mathbb{R}) & \rightarrow L\left(H^{s-2}(\mathbb{R}), H^{s}(\mathbb{R})\right) \\
\varphi & \mapsto\left[v \mapsto R_{\varphi}\left(1-\partial_{x}^{2}\right)^{-1} R_{\varphi^{-1}} v\right]
\end{aligned}
$$

is analytic. Piecing the maps together we get from the identity

$$
\begin{aligned}
\Gamma_{\varphi}(u, v) & =R_{\varphi}\left(1-\partial_{x}^{2}\right)^{-1} B\left(R_{\varphi^{-1}} u, R_{\varphi^{-1}} v\right) \\
& =R_{\varphi}\left(1-\partial_{x}^{2}\right)^{-1} R_{\varphi^{-1}} R_{\varphi} B\left(R_{\varphi^{-1}} u, R_{\varphi^{-1}} u\right)
\end{aligned}
$$

that $\Gamma: \mathscr{D}^{s}(\mathbb{R}) \rightarrow L\left(H^{s}(\mathbb{R}), H^{s}(\mathbb{R}) ; H^{s}(\mathbb{R})\right)$ is analytic. This completes the proof of the theorem.

Now consider the initial value problem (IVP)

$$
\left\{\begin{array}{c}
\varphi_{t t}=\Gamma_{\varphi}\left(\varphi_{t}, \varphi_{t}\right) \\
\varphi(0)=i d \in \mathscr{D}^{s}(\mathbb{R}), \quad \varphi_{t}(0)=u_{0} \in H^{s}(\mathbb{R}) .
\end{array}\right.
$$

The Picard theorem gives us local solutions to the IVP (3.2). With this and the discussion at the and of section 2 we get the following local existence result.

Lemma 3.2. Let $s>3 / 2$. Given $u_{0} \in H^{s}(\mathbb{R})$, there exists $u \in C^{0}\left([0, T], H^{s}(\mathbb{R})\right)$, for some $T>0$, such that

$$
u(t)=u_{0}+\int_{0}^{t}\left(1-\partial_{x}^{2}\right)^{-1}\left(-b u u_{x}+(b-3) u_{x} u_{x x}\right)-u u_{x}
$$

holds for all $t \in[0, T]$.

Proof. Consider the IVP (3.2). Since $\Gamma$ is smooth, there exists $T>0$, such that we have a

$$
\varphi \in C^{\infty}\left([0, T], \mathscr{D}^{s}(\mathbb{R})\right)
$$

solving (3.2). We claim that $u:=\varphi_{t} \circ \varphi^{-1}$ is a solution to (3.3). From [23] we know that $u \in$ $C^{0}\left([0, T], H^{s}(\mathbb{R})\right)$. We also have $u(0)=\varphi_{t}(0)=u_{0}$. From the Sobolev imbedding theorem we see 
that $u$ resp. $\varphi$ are in $C^{1}([0, T] \times \mathbb{R})$. Taking the $t$-derivative of $u \circ \varphi$ we get

$$
u_{t} \circ \varphi+u_{x} \circ \varphi \cdot \varphi_{t}=u_{t} \circ \varphi+u_{x} \circ \varphi \cdot u \circ \varphi .
$$

On the other hand we have

$$
(u \circ \varphi)_{t}=\varphi_{t t}=\Gamma_{\varphi}\left(\varphi_{t}, \varphi_{t}\right)
$$

as functions on $[0, T] \times \mathbb{R}$. Thus we get

$$
u_{t} \circ \varphi+u_{x} \circ \varphi \cdot u \circ \varphi=\Gamma_{\varphi}\left(\varphi_{t}, \varphi_{t}\right)
$$

or

$$
\begin{aligned}
u_{t} & =\Gamma_{\varphi}\left(\varphi_{t}, \varphi_{t}\right) \circ \varphi^{-1}-u u_{x} \\
& =\left(1-\partial_{x}^{2}\right)^{-1}\left(-b u u_{x}+(b-3) u_{x} u_{x x}\right)-u u_{x}
\end{aligned}
$$

as functions on $[0, T] \times \mathbb{R}$. As both sides are continuous functions, we get by the fundamental lemma of calculus for $t \in[0, T]$

$$
u(t)=u_{0}+\int_{0}^{t}\left(1-\partial_{x}^{2}\right)^{-1}\left(-b u u_{x}+(b-3) u_{x} u_{x x}\right)-u u_{x} .
$$

But as the integrand is in $C^{0}\left([0, T], H^{s-1}(\mathbb{R})\right)$, the identity (3.4) holds also in $H^{s-1}(\mathbb{R})$.

To get uniqueness we use the fact that for $u \in C\left([0, T] ; H^{s}(\mathbb{R})\right)$ there is a unique flow $\varphi \in$ $C^{1}\left([0, T] ; \mathscr{D}^{s}(\mathbb{R})\right)$, i.e. $\varphi$ solving

$$
\varphi_{t}=u \circ \varphi, \quad \varphi(0)=i d
$$

- see [24]. With this we will prove

Lemma 3.3. Let $s>3 / 2$. Assume that we have two solutions $u, w \in C^{0}\left([0, T], H^{s}(\mathbb{R})\right)$ to the Cauchy problem (1.3) with $u(0)=w(0)=u_{0} \in H^{s}(\mathbb{R})$. Then we have actually $u=w$ on $[0, T]$.

Proof. Consider the flows $\varphi$ resp. $\psi$ in $C^{1}\left([0, T], \mathscr{D}^{s}(\mathbb{R})\right)$ corresponding to $u$ resp. $w$ as discussed above. We will show that $\varphi$ resp. $\psi$ are geodesics. By the uniqueness of geodesics with the same initial condition we will get $\varphi=\psi$ resp. $\varphi_{t} \circ \varphi^{-1}=\psi_{t} \circ \psi^{-1}$ or equivalently $u=w$. Now consider $u \circ \varphi$ which is $C^{1}$ in $[0, T] \times \mathbb{R}$. Taking the $t$-derivative we get pointwise

$$
\frac{d}{d t}(u \circ \varphi)=u_{t} \circ \varphi+u_{x} \circ \varphi \cdot u \circ \varphi
$$

Since $u$ is a solution of the Cauchy problem we have

$$
\begin{aligned}
\frac{d}{d t}(u \circ \varphi) & =R_{\varphi}\left(\left(1-\partial_{x}^{2}\right)^{-1}\left(-b u u_{x}+(b-3) u_{x} u_{x} x\right)\right) \\
& =\Gamma_{\varphi}\left(\varphi_{t} \circ \varphi^{-1}, \varphi_{t} \circ \varphi^{-1}\right)
\end{aligned}
$$

where $\Gamma$ is as in (2.4). From the fundamental lemma of calculus we get

$$
\varphi_{t}(t)=u_{0}+\int_{0}^{t} \Gamma_{\varphi}\left(\varphi_{t} \circ \varphi^{-1}, \varphi_{t} \circ \varphi^{-1}\right) .
$$

But as the integrand is in $C^{0}\left([0, T], H^{s}(\mathbb{R})\right)$ we see that $\varphi \in C^{2}\left([0, T], \mathscr{D}^{s}(\mathbb{R})\right)$ and that it is a geodesic. Hence the claim. 
Using Lemma 3.2 and Lemma 3.3 we get

Theorem 3.2. Let $s>3 / 2$. The Cauchy problem (1.3) is locally well-posed in $H^{s}(\mathbb{R})$, i.e. given $u_{0} \in H^{s}(\mathbb{R})$ there exists a solution $u \in C^{0}\left([0, T], H^{s}(\mathbb{R})\right)$ for some $T>0$. Further $u$ is unique on $[0, T]$ and the correspondence $u_{0} \rightarrow u$ is continuous.

Remark 3.1. The correspondence $u_{0} \rightarrow u$ is meant as a map

$$
U_{T} \rightarrow C^{0}\left([0, T], H^{s}(\mathbb{R})\right), \quad u_{0} \mapsto u
$$

This result is not new. This was e.g. done in [29]. They looked at a regularized version of (3.1) and took the limit. Also in [31] there is a similar result. They use Kato's abstract semigroup method. The same method is also used in [13] for the periodic case. We used the geometric setting as was e.g. done in [7] to achieve local well-posedness via the Picard theorem.

\section{Non-uniform dependence of the solution map}

In this section we will prove the non-uniform dependence of the solution map, i.e. we will prove Theorem 1.2. Recall that we denoted for $T>0$ the set $U_{T} \subseteq H^{s}(\mathbb{R})$ to be those $u_{0}$ for which we have existence beyond $T$. Note also that we have the following scaling property for equation (1.1): If $u$ is a solution then for $\lambda>0$

$$
v(x, t):=\lambda u(x, \lambda t)
$$

is also a solution and $U_{\lambda T}=\frac{1}{\lambda} U_{T}$. Therefore it suffices to consider the case $T=1$ to prove Theorem 1.2. Hence the theorem will follow from

Proposition 4.1. Let $s>3 / 2$ and $U:=\left.U_{T}\right|_{T=1}$. Denote by $\Phi$ the time-one map $\Phi: U \rightarrow$ $H^{s}(\mathbb{R}), u_{0} \mapsto u(1)$ for the Cauchy problem (1.1). Then $\Phi$ is nowhere locally uniformly continuous, i.e. for any non-empty $V \subseteq U$ the restriction $\left.\Phi\right|_{V}$ is not uniformly continuous.

To prove Proposition 4.1 we will use a conserved quantity (cf [17], Proposition 9). Consider equation (1.2) and let $u$ be a solution, $\varphi$ its corresponding flow. Then we have, omitting the arguments $(t, x)$,

Lemma 4.2. Let $y:=\left(1-\partial_{x}^{2}\right) u$. Then we have for all the following identity in $H^{s-2}(\mathbb{R})$

$$
y \circ \varphi \cdot\left(\varphi_{x}\right)^{b}=y(t=0)
$$

Proof. Taking the $t$-derivate of $y \circ \varphi$ we get

$$
\frac{d}{d t} y \circ \varphi=\varphi_{t t}-\frac{\varphi_{t t x x}}{\varphi_{x}^{2}}+2 \frac{\varphi_{t x x} \cdot \varphi_{t x}}{\varphi_{x}^{3}}+\frac{\varphi_{t t x} \cdot \varphi_{x x}}{\varphi_{x}^{3}}+\frac{\varphi_{t x} \cdot \varphi_{t x x}}{\varphi_{x}^{3}}-3 \frac{\varphi_{t x} \cdot \varphi_{x x} \cdot \varphi_{t x}}{\varphi_{x}^{4}}
$$

On the other hand equation (3.2) gives

$$
R_{\varphi}\left(1-\partial_{x}^{2}\right)\left(\varphi_{t t} \circ \varphi^{-1}\right)=R_{\varphi}\left(-b\left(\varphi_{t} \circ \varphi^{-1}\right) \cdot\left(\varphi_{t} \circ \varphi^{-1}\right)_{x}+(b-3)\left(\varphi_{t} \circ \varphi^{-1}\right)_{x} \cdot\left(\varphi_{t} \circ \varphi^{-1}\right)_{x x}\right)
$$

Expanding this equation we get

$$
\varphi_{t t}-\frac{\varphi_{t t x x}}{\varphi_{x}^{2}}+\frac{\varphi_{t t x} \cdot \varphi_{x x}}{\varphi_{x}^{3}}=-b \frac{\varphi_{t} \cdot \varphi_{t x}}{\varphi_{x}}+(b-3) \frac{\varphi_{t x}}{\varphi_{x}}\left(\frac{\varphi_{t x x}}{\varphi_{x}^{2}}-\frac{\varphi_{t x} \cdot \varphi_{x x}}{\varphi_{x}^{3}}\right)
$$


Hence from (4.2)

$$
\begin{aligned}
\frac{d}{d t}\left[\left(R_{\varphi} y\right) \cdot \varphi_{x}^{b}\right]=\left[\varphi_{t t}-\frac{\varphi_{t t x x}}{\varphi_{x}^{2}}+3 \frac{\varphi_{t x x} \cdot \varphi_{t x}}{\varphi_{x}^{3}}+\frac{\varphi_{t t x} \cdot \varphi_{x x}}{\varphi_{x}^{3}}\right. & \left.-3 \frac{\varphi_{t x}^{2} \cdot \varphi_{x x}}{\varphi_{x}^{4}}\right] \cdot \varphi_{x}^{b} \\
& +\left[\varphi_{t}-\frac{\varphi_{t x x}}{\varphi_{x}^{2}}+\frac{\varphi_{t x} \cdot \varphi_{x x}}{\varphi_{x}^{3}}\right] \cdot b \varphi_{x}^{b-1} \varphi_{t x}
\end{aligned}
$$

Combining (4.4) and (4.3) we get

$$
\frac{d}{d t}\left[\left(R_{\varphi} y\right) \cdot \varphi_{x}^{b}\right]=0
$$

hence $[0, T] \rightarrow H^{s-2}(\mathbb{R}), t \mapsto y \circ \varphi \cdot \varphi_{x}^{b}$ is constant, i.e. (4.1) holds.

As $1-\partial_{x}^{2}: H^{s}(\mathbb{R}) \rightarrow H^{s-2}(\mathbb{R})$ is an isomorphism, it will be enough to establish that $y_{0} \mapsto y(1)$ is nowhere locally uniformly continuous in order to show Proposition 4.1. We will use (4.1) in the form

$$
y(1)=\left(\frac{y_{0}}{\varphi_{x}(1)^{b}}\right) \circ \varphi(1)^{-1}
$$

The approach is as in [26]. The idea is to produce a slight change $\tilde{\varphi}(1)$ so that $\tilde{y}_{0}$ doesn't change much, but $\tilde{y}(1)$ does. Since composition behaves bad this works. To make such perturbations we will employ the properties of the exponential map. But first we have to establish some preleminary lemmas.

Lemma 4.3. Given $\varphi_{\bullet} \in \mathscr{D}^{s}(\mathbb{R})$ there exists a neighborhood $W$ of $\varphi_{\bullet}$ in $\mathscr{D}^{s}(\mathbb{R})$, such that for some constant $C>0$ we have

$$
\frac{1}{C}\|y\|_{s-2} \leq\left\|R_{\varphi}^{-1}\left(y / \varphi_{x}^{b}\right)\right\|_{s-2} \leq C|| y \|_{s-2}
$$

for all $y \in H^{s-2}(\mathbb{R})$ and $\varphi \in W$.

Proof. First we establish the second inequality. For $\varphi_{n} \rightarrow \varphi_{\bullet}$ in $\mathscr{D}^{s}(\mathbb{R})$ we have (see Remark A.2) $R_{\varphi_{n}}^{-1}\left(y / \varphi_{x}^{b}\right)$ converges weakly to $y$ in $H^{s-2}(\mathbb{R})$. By the uniform boundedness principle we get a neighborhood $W_{1}$ of $\varphi_{\bullet}$ in $\mathscr{D}^{s}(\mathbb{R})$ and $C_{1}>0$ such that

$$
\left\|R_{\varphi}^{-1}\left(y / \varphi_{x}^{b}\right)\right\|_{s-2} \leq C|| y \|_{s-2}
$$

for all $\varphi \in W_{1}$ and $y \in H^{s-2}(\mathbb{R})$. Now consider the first inequality. As in the first part we get a $W_{2}$ and $C_{2}>0$ with

$$
\left\|\varphi_{x}^{b} R_{\varphi} y\right\|_{s-2} \leq C_{2}\|y\|_{s-2}
$$

for all $\varphi \in W_{2}$ and $y \in H^{s-2}(\mathbb{R})$. Taking for $y$ the expression $R_{\varphi}^{-1}\left(y / \varphi_{x}^{b}\right)$ gives the first inequalty.

Lemma 4.4. Let $s>3 / 2$. The set of $u_{0} \in U \cap H^{s+1}$ with $d_{u_{0}} \exp \neq 0$ is dense in $U$.

Proof. As $d_{0}$ exp is the identity map and $u_{0} \mapsto d_{u_{0}}$ exp is analytic the claim follows immediately.

Now we can give a proof for Proposition 4.1 
Proof of Proposition 4.1. Take $u_{0} \in U$. We will show that $\Phi$ is not uniformly continuous on any neighborhood of $u_{0}$. As easily seen we can restrict ourselves to a dense subset of $U$. So we can assume $u_{0} \in H^{s+1}$ and $d_{u_{0}} \exp \neq 0$ by Lemma 4.4. In particular we can fix $v \in H^{s}(\mathbb{R}) \backslash\{0\}$ and $x_{0} \in \mathbb{R}$ with

$$
\left(d_{u_{0}} \exp (v)\right)\left(x_{0}\right)>m\|v\|_{s}, \quad m>0
$$

First we choose $R_{1}>0$ such that Lemma 4.3 holds for $\varphi_{\bullet}=\exp \left(u_{0}\right)$ in the ball $B_{R_{1}}\left(u_{0}\right)$, i.e.

$$
\frac{1}{C_{1}}\|y\|_{s-2} \leq\left\|R_{\varphi}^{-1}\left(y / \varphi_{x}^{b}\right)\right\|_{s-2} \leq C_{1}\|y\|_{s-2}
$$

for all $y \in H^{s-2}(\mathbb{R})$ and $\varphi \in B_{R_{1}}\left(u_{0}\right)$. Taking $R_{2} \leq R_{1}$ we can ensure additionally

$$
\left\|R_{\varphi}^{-1} y\right\|_{s-2} \leq C_{2}\|y\|_{s-2}
$$

for all $y \in H^{s-2}(\mathbb{R})$ and $\varphi \in B_{R_{2}}\left(u_{0}\right)$. By choosing $R_{3} \leq R_{2}$ we can establish the conditions of Lemma A.5 and A.6 for all $\varphi \in B_{R_{3}}$ where in the following we denote the constant appearing in both lemmas by $C_{3}$. Further we denote by $C>0$ the constant from the Sobolev imbedding

$$
\|f\|_{\infty} \leq C\|f\|_{s}
$$

Take arbitrary $w, h \in H^{s}(\mathbb{R})$ with $w, w+h \in B_{R_{3}}\left(u_{0}\right)$ and consider the Taylor expansion

$$
\exp (w+h)=\exp (w)+d_{w} \exp (w)+\int_{0}^{1}(1-t) d_{w+t h}^{2} \exp (h, h) d t
$$

Choosing $0<R_{4} \leq R_{3}$ we can garantuee

$$
\begin{gathered}
\left\|d_{w}^{2} \exp (h, h)\right\|_{s} \leq K\|h\|_{s}^{2} \\
\left\|d_{w_{1}}^{2} \exp (h, h)-d_{w_{2}}^{2} \exp (h, h)\right\|_{s} \leq K\left\|w_{1}-w_{2}\right\|\left\|_{s}\right\| h \|_{s}^{2}
\end{gathered}
$$

for all $w, w_{1}, w_{2} \in B_{R_{4}}\left(u_{0}\right)$ and some constant $K>0$. By further decreasing $R_{5} \leq R_{4}$ we can ensure $\max \left\{C \cdot K \cdot R_{5}, C \cdot K \cdot R_{5}^{2}\right\}<m / 2$. Finally by choosing $R_{*} \leq R_{5}$ we can ensure

$$
|\varphi(x)-\varphi(y)| \leq L|x-y|
$$

for all $\varphi \in \exp \left(B_{R_{*}}\left(u_{0}\right)\right)$. The goal is now to prove that $\Phi$ is not uniformly continuous on $B_{R}\left(u_{0}\right)$ for $0<R \leq R_{*}$. So we fix $R \leq R_{*}$. In order to apply Lemma B.2 resp. Lemma B.3 we define the sequence of numbers

$$
r_{n}=\frac{m}{8 n}\|v\|_{s}, \quad n \geq 1
$$

and choose $w_{n} \in C_{c}^{\infty}(\mathbb{R})$ with support in $\left(x_{0}-\frac{1}{L} r_{n}, x_{0}+\frac{1}{L} r_{n}\right)$ and $\left\|w_{n}\right\|_{s}=R / 4$. Further we define $v_{n}:=v / n$ and let $N \geq 1$ such that $\left\|v_{n}\right\|_{s} \leq R / 4$ for $n \geq N$. With this preliminary work we define for 
$n \geq N$ two sequences of initial data:

$$
x_{n}=u_{0}+w_{n} \quad \text { and } \quad \tilde{x}_{n}=x_{n}+v_{n}=u_{0}+w_{n}+v_{n}
$$

We clearly have $x_{n}, y_{n} \in B_{R}\left(u_{0}\right)$ for $n \geq N$ and $\left\|x_{n}-\tilde{x}_{n}\right\|_{s} \rightarrow 0$ for $n \rightarrow \infty$. Correspondingly we define

$$
\varphi_{n}=\exp \left(x_{n}\right) \quad \text { and } \quad \tilde{\varphi}_{n}=\exp \left(\tilde{x}_{n}\right)
$$

We claim that $\limsup _{n \rightarrow \infty}\left\|\Phi\left(x_{n}\right)-\Phi\left(\tilde{x}_{n}\right)\right\|_{s}>0$. With $y_{n}=\left(1-\partial_{x}^{2}\right) x_{n}$ and $\tilde{y}_{n}=\left(1-\partial_{x}^{2}\right) \tilde{x}_{n}$ and using the conservation law (4.5) it is enough to prove

$$
\limsup _{n \rightarrow \infty}\left\|R_{\varphi_{n}}^{-1}\left(y_{n} /\left(\varphi_{n}\right)_{x}^{b}\right)-R_{\tilde{\varphi}_{n}}^{-1}\left(\tilde{y}_{n} /\left(\tilde{\varphi}_{n}\right)_{x}^{b}\right)\right\|_{s-2}>0
$$

We consider the parts of $y_{n}, \tilde{y}_{n}$ seperately

$$
y_{n}=\left(1-\partial_{x}^{2}\right)\left(u_{0}+w_{n}\right) \quad \text { and } \quad \tilde{y}_{n}=\left(1-\partial_{x}^{2}\right)\left(u_{0}+w_{n}+v_{n}\right)
$$

For the $u_{0}$-part we have, denoting $y_{0}=\left(1-\partial_{x}^{2}\right) u_{0} \in H^{s-1}$,

$$
\begin{aligned}
\| R_{\varphi_{n}}^{-1}\left(y_{0} /\left(\varphi_{n}\right)_{x}^{b}\right)-R_{\tilde{\varphi}_{n}}^{-1}\left(y_{0} /\left(\tilde{\varphi}_{n}\right)_{x}^{b}\left\|_{s-2} \leq\right\| R_{\varphi_{n}}^{-1}\left(y_{0} /\left(\varphi_{n}\right)_{x}^{b}\right)-R_{\varphi_{n}}^{-1}\left(y_{0} /\left(\tilde{\varphi}_{n}\right)_{x}^{b}\right) \|_{s-2}\right. \\
+\left\|R_{\varphi_{n}}^{-1}\left(y_{0} /\left(\tilde{\varphi}_{n}\right)_{x}^{b}\right)-R_{\tilde{\varphi}_{n}}^{-1}\left(y_{0} /\left(\tilde{\varphi}_{n}\right)_{x}^{b}\right)\right\|_{s-2}
\end{aligned}
$$

The first term on the right can be estimated by

$$
\left\|R_{\varphi_{n}}^{-1}\left(y_{0} /\left(\varphi_{n}\right)_{x}^{b}\right)-R_{\varphi_{n}}^{-1}\left(y_{0} /\left(\tilde{\varphi}_{n}\right)_{x}^{b}\right)\right\|_{s-2} \leq C_{2}|| y_{0} /\left(\varphi_{n}\right)_{x}^{b}-y_{0} /\left(\tilde{\varphi}_{n}\right)_{x}^{b} \|_{s-2}
$$

The latter goes to 0 as $n \rightarrow \infty$ as dividing by $\varphi_{x}^{b}$ is an analytic process. For the second term we have

$$
\begin{aligned}
\| R_{\varphi_{n}}^{-1}\left(y_{0} /\left(\tilde{\varphi}_{n}\right)_{x}^{b}\right)-R_{\tilde{\varphi}_{n}}^{-1}\left(y_{0} /\left(\tilde{\varphi}_{n}\right)_{x}^{b} \|_{s-2}\right. & \leq C_{3}\left\|y_{0} /\left(\tilde{\varphi}_{n}\right)_{x}^{b}\right\|_{s-1}\left\|\varphi_{n}^{-1}-\tilde{\varphi}_{n}^{-1}\right\|_{s-1} \\
& \leq\left. C_{3}^{2}\left\|y_{0} /\left(\tilde{\varphi}_{n}\right)_{x}^{b}\right\|_{s-1}\left\|\varphi_{n}-\tilde{\varphi}_{n}\right\|\right|_{s}
\end{aligned}
$$

which goes to 0 as $y_{0} /\left(\tilde{\varphi}_{n}\right)_{x}^{b}$ is bounded in $H^{s-1}$. For the $v_{n}$-term we have

$$
\left\|R_{\tilde{\varphi}_{n}}^{-1}\left(\left(1-\partial_{x}^{2}\right) v_{n} /\left(\tilde{\varphi}_{n}\right)_{x}^{b}\right)\right\|_{s-2} \leq C_{2}\left\|\left(1-\partial_{x}^{2}\right) v_{n} /\left(\tilde{\varphi}_{n}\right)_{x}^{b}\right\|_{s-2}
$$

which by the definition of $v_{n}$ goes to zero. Hence we conclude

$$
\begin{aligned}
\limsup _{n \rightarrow \infty} \| R_{\varphi_{n}}^{-1}\left(y_{n} /\left(\varphi_{n}\right)_{x}^{b}\right) & -R_{\tilde{\varphi}_{n}}^{-1}\left(\tilde{y}_{n} /\left(\tilde{\varphi}_{n}\right)_{x}^{b}\right) \|_{s-2} \\
& =\limsup _{n \rightarrow \infty}\left\|R_{\varphi_{n}}^{-1}\left(\left(1-\partial_{x}^{2}\right) w_{n} /\left(\varphi_{n}\right)_{x}^{b}\right)-R_{\tilde{\varphi}_{n}}^{-1}\left(\left(1-\partial_{x}^{2}\right) w_{n} /\left(\tilde{\varphi}_{n}\right)_{x}^{b}\right)\right\|_{s-2}
\end{aligned}
$$

The claim is now that the latter two terms have disjoint support. To establish this we estimate $\left|\varphi_{n}\left(x_{0}\right)-\tilde{\varphi}_{n}\left(x_{0}\right)\right|$. By the Taylor expansion we have

$$
\varphi_{n}=\exp \left(u_{0}\right)+d_{u_{0}} \exp \left(w_{n}\right)+\int_{0}^{1}(1-t) d_{u_{0}+t w_{n}}^{2}\left(w_{n}, w_{n}\right) d t
$$

and similarly

$$
\tilde{\varphi}_{n}=\exp \left(u_{0}\right)+d_{u_{0}} \exp \left(w_{n}+v_{n}\right)+\int_{0}^{1}(1-t) d_{u_{0}+t\left(w_{n}+v_{n}\right)}^{2}\left(w_{n}+v_{n}, w_{n}+v_{n}\right) d t
$$


For the latter quadratic term we have

$$
d_{u_{0}+t\left(w_{n}+v_{n}\right)}^{2}\left(w_{n}+v_{n}, w_{n}+v_{n}\right)=d_{u_{0}+t\left(w_{n}+v_{n}\right)}^{2}\left(w_{n}, w_{n}\right)+2 d_{u_{0}+t\left(w_{n}+v_{n}\right)}^{2}\left(w_{n}, v_{n}\right)+d_{u_{0}+t\left(w_{n}+v_{n}\right)}^{2}\left(v_{n}, v_{n}\right)
$$

Thus we can write

$$
\varphi_{n}-\tilde{\varphi}_{n}=d_{u_{0}} \exp \left(v_{n}\right)+\mathscr{R}_{1}+\mathscr{R}_{2}+\mathscr{R}_{3}
$$

where

$$
\mathscr{R}_{1}=\int_{0}^{1}(1-t)\left(d_{u_{0}+t\left(w_{n}+v_{n}\right)}^{2}\left(w_{n}, w_{n}\right)-d_{u_{0}+t\left(w_{n}+v_{n}\right)}^{2}\left(w_{n}, w_{n}\right)\right) d t
$$

and

$$
\mathscr{R}_{2}=2 \int_{0}^{1}(1-t) d_{u_{0}+t\left(w_{n}+v_{n}\right)}^{2}\left(w_{n}, v_{n}\right) d t
$$

and

$$
\mathscr{R}_{3}=\int_{0}^{1}(1-t) d_{u_{0}+t\left(w_{n}+v_{n}\right)}^{2}\left(v_{n}, v_{n}\right) d t
$$

For these we have

$$
\left\|\mathscr{R}_{1}\right\|_{\infty} \leq C \mid\left\|\mathscr{R}_{1}\right\|_{s} \leq C K\left\|v_{n}\right\|\left\|_{s}\right\| w_{n}\left\|_{s}^{2} \leq \frac{1}{n} C K\right\| v\left\|_{s}(R / 4)^{2} \leq \frac{1}{4 n} C K R^{2}\right\| v \|_{s}
$$

and

$$
\left\|\mathscr{R}_{2}\right\|_{\infty} \leq C|| \mathscr{R}_{2}\left\|_{s} \leq 2 C K|| v_{n}\right\|\left\|_{s}\right\| w_{n}\left\|_{s} \leq \frac{1}{n} C K\right\| v\left\|_{s}(R / 4) \leq \frac{2}{4 n} C K R\right\| v \|_{s}
$$

and

$$
\left\|\mathscr{R}_{3}\right\|_{\infty} \leq C\left\|\mathscr{R}_{3}\right\|_{s} \leq C K\left\|v_{n}\right\|_{s}^{2} \leq \frac{1}{n} C K\|v\|_{s}(R / 4) \leq \frac{1}{4 n} C K R\|v\|_{s}
$$

Therefore

$$
\begin{aligned}
\left|\varphi\left(x_{0}\right)-\tilde{\varphi}\left(x_{0}\right)\right| & \geq\left|d_{u_{0}} \exp \left(v_{n}\right)\right|-\left\|\mathscr{R}_{1}\right\|_{\infty}-\left\|\mathscr{R}_{2}\right\|_{\infty}-\left\|\mathscr{R}_{3}\right\|_{\infty} \\
& \geq \frac{1}{n} m\|v\|_{s}-\frac{1}{n} \frac{m}{2}\|v\|_{s}=\frac{m}{2 n}\|v\|_{s}
\end{aligned}
$$

The support of $R_{\varphi_{n}}^{-1}\left(\left(1-\partial_{x}^{2}\right) w_{n} /\left(\varphi_{n}\right)_{x}^{b}\right)$ is contained in $\left(\varphi_{n}\left(x_{0}\right)-r_{n}, \varphi_{n}\left(x_{0}\right)+r_{n}\right)$ taking into account the lipschitz property of $\varphi_{n}$ with lipschitz constant $L$ and the definition of $w_{n}$. Analogously the support of $R_{\tilde{\varphi}_{n}}^{-1}\left(\left(1-\partial_{x}^{2}\right) w_{n} /\left(\tilde{\varphi}_{n}\right)_{x}^{b}\right)$ is contained in $\left(\tilde{\varphi}_{n}\left(x_{0}\right)-r_{n}, \tilde{\varphi}_{n}\left(x_{0}\right)+r_{n}\right)$. Note that the conditions 
of Lemma B. 2 resp. B.3 are fullfilled (with $s-2$ playing the role of $s$ in the Lemma) as

$$
r_{n} \leq\left|\varphi_{n}\left(x_{0}\right)-\tilde{\varphi}_{n}\left(x_{0}\right)\right| / 4
$$

Thus we have

$$
\begin{aligned}
& \limsup _{n \rightarrow \infty} \| R_{\varphi_{n}}^{-1}\left(\left(1-\partial_{x}^{2}\right) w_{n} /\left(\varphi_{n}\right)_{x}^{b}\right)-R_{\tilde{\varphi}_{n}}^{-1}\left(\left(1-\partial_{x}^{2}\right) w_{n} /\left(\tilde{\varphi}_{n}\right)_{x}^{b}\right) \|_{s-2}^{2} \\
& \geq \limsup _{n \rightarrow \infty} \tilde{C}\left(\left\|R_{\varphi_{n}}^{-1}\left(\left(1-\partial_{x}^{2}\right) w_{n} /\left(\varphi_{n}\right)_{x}^{b}\right)\right\|_{s-2}^{2}+\left\|R_{\tilde{\varphi}_{n}}^{-1}\left(\left(1-\partial_{x}^{2}\right) w_{n} /\left(\tilde{\varphi}_{n}\right)_{x}^{b}\right)\right\|_{s-2}^{2}\right) \\
& \quad \geq \limsup _{n \rightarrow \infty} \tilde{C} \frac{2}{C^{2}}\left\|\left(1-\partial_{x}^{2}\right) w_{n}\right\|_{s-2}^{2} \geq \limsup _{n \rightarrow \infty} \tilde{K}\left\|w_{n}\right\|_{s}^{2}=\tilde{K} R^{2} / 4
\end{aligned}
$$

So for any $R \leq R_{*}$ we have constructed $\left(x_{n}\right)_{n \geq 1},\left(\tilde{x}_{n}\right)_{n \geq 1} \subseteq B_{R}\left(u_{0}\right)$ with $\lim _{n \rightarrow \infty}\left\|x_{n}-\tilde{x}_{n}\right\|_{s}=0$ and $\limsup _{n \rightarrow \infty}|| \Phi\left(x_{n}\right)-\Phi\left(\tilde{x}_{n}\right) \|_{s} \geq C \cdot R$ for some constant $C>0$ independent of $R$ showing the claim.

\section{Appendix A. Sobolev spaces with negative indices}

In this section we derive the formulas for the expressions which involve Sobolev spaces with negative indices.

Lemma A.1. Let $1 / 2<s_{1}<1$ and $-1 / 2<s_{2}<0$. Then multiplication

$$
H^{s_{1}}(\mathbb{R}) \times H^{s_{1}}(\mathbb{R}) \rightarrow H^{s_{1}}(\mathbb{R}), \quad(f, g) \mapsto f \cdot g
$$

extends to a continuous map

$$
H^{s_{1}}(\mathbb{R}) \times H^{s_{2}}(\mathbb{R}) \rightarrow H^{s_{2}}(\mathbb{R})
$$

where $H^{s_{2}}(\mathbb{R})$ denotes the dual of $H^{-s_{2}}(\mathbb{R})$.

Proof. For $f \in H^{s_{1}}(\mathbb{R}), g \in H^{s_{2}}(\mathbb{R})$ we define $f \cdot g \in H^{s_{2}}(\mathbb{R})$ by its action on a testfunction $\psi$ as

$$
\langle f \cdot g, \psi\rangle:=\langle g, f \cdot \psi\rangle
$$

where $\langle\cdot, \cdot\rangle$ denotes the duality pairing of $H^{s_{2}}(\mathbb{R})$ and $H^{-s_{2}}(\mathbb{R})$. As $f \cdot \psi \in H^{-s_{2}}(\mathbb{R})$ this definition makes sense. Further we have

$$
|\langle g, f \cdot \psi\rangle| \leq\|g\|_{s_{2}}\|f \cdot \psi\|_{-s_{2}} \leq C\|g\|_{s_{2}}\|f\|_{s_{1}}\|\psi\|_{-s_{2}}
$$

where we have used that multiplication

$$
H^{s_{1}}(\mathbb{R}) \times H^{-s_{2}}(\mathbb{R}) \rightarrow H^{-s_{2}}(\mathbb{R})
$$

is continuous - see e.g. [23]. This shows that $f \cdot g \in H^{s_{2}}(\mathbb{R})$. But it also shows that

$$
\|f \cdot g\|_{s_{2}} \leq C|| f||_{s_{1}}|| g \|_{s_{2}} .
$$

Hence the claim. 
For the product we have the following Leibniz rule.

Lemma A.2. Let $1 / 2<\tilde{s}<1$ and $f, g \in H^{\tilde{s}}(\mathbb{R})$. Then $f \cdot g \in H^{\tilde{s}}(\mathbb{R})$ with

$$
\partial_{x}(f \cdot g)=f_{x} \cdot g+f \cdot g_{x}
$$

where the subscript refers to differentiation.

Proof. We just have to prove the formula for the derivative. For test functions $\psi, \phi$ we have

$$
\begin{aligned}
\left\langle\partial_{x}(f \cdot \phi), \psi\right\rangle & =-\left\langle f \cdot \phi, \psi_{x}\right\rangle=-\left\langle f, \phi \cdot \psi_{x}\right\rangle=-\left\langle f,(\phi \cdot \psi)_{x}-\phi_{x} \cdot \psi\right\rangle \\
& =\left\langle\phi \cdot f_{x}, \psi\right\rangle+\left\langle f \cdot \phi_{x}, \psi\right\rangle .
\end{aligned}
$$

Therefore we have the following identity in $H^{\tilde{s}-1}(\mathbb{R})$

$$
\partial_{x}(f \cdot \phi)=f_{x} \cdot \phi+f \cdot \phi_{x} .
$$

Now letting $\phi$ tend to $g$ in $H^{1-\tilde{s}}(\mathbb{R})$ we get by Lemma A.1

$$
\partial_{x}(f \cdot g)=f_{x} \cdot g+f \cdot g_{x}
$$

as elements in $H^{\tilde{s}-1}(\mathbb{R})$.

Now we extend right translation $R_{\varphi}$ to negative Sobolev spaces

Lemma A.3. Let $s>3 / 2$ and $-1 / 2<\tilde{s}<0$. For $\varphi \in \mathscr{D}^{s}(\mathbb{R})$ the map

$$
R_{\varphi}: H^{s}(\mathbb{R}) \rightarrow H^{s}(\mathbb{R}), \quad f \mapsto f \circ \varphi
$$

extends to a continuous map $H^{\tilde{s}}(\mathbb{R}) \rightarrow H^{\tilde{s}}(\mathbb{R})$.

Proof. Let $f \in H^{\tilde{s}}(\mathbb{R})$ ans $\psi$ a testfunction. We define

$$
\left\langle R_{\varphi} f, \psi\right\rangle:=\left\langle f, \frac{\psi \circ \varphi^{-1}}{\varphi_{x} \circ \varphi^{-1}}\right\rangle .
$$

We know - see e.g. [23] - that

$$
\left\|\frac{\psi \circ \varphi^{-1}}{\varphi_{x} \circ \varphi^{-1}}\right\|_{-\tilde{s}} \leq C|| \psi \|_{-\tilde{s}}
$$

holds. Therefore $R_{\varphi} f \in H^{\tilde{s}}(\mathbb{R})$ and further

$$
\left|\left\langle R_{\varphi} f, \psi\right\rangle\right|=\left|\left\langle f, \frac{\psi \circ \varphi^{-1}}{\varphi_{x} \circ \varphi^{-1}}\right\rangle\right| \leq C|| f\left\|_{\tilde{s}}\right\| \psi \|_{-\tilde{s}}
$$

which shows that $R_{\varphi}: H^{\tilde{s}}(\mathbb{R}) \rightarrow H^{\tilde{s}}(\mathbb{R})$ is a continuous linear map.

Remark A.1. We will sometimes write $f \circ \varphi$ instead of $R_{\varphi} f$ even if $f$ is in a negative Sobolev space. 
Remark A.2. The composition map

$$
H^{\tilde{s}}(\mathbb{R}) \times \mathscr{D}^{s}(\mathbb{R}) \rightarrow H^{\tilde{s}}(\mathbb{R}), \quad(f, \varphi) \mapsto f \circ \varphi
$$

is not continuous. But as can be seen from (A.1) it is weakly continuous, i.e

$$
H^{\tilde{s}}(\mathbb{R}) \times \mathscr{D}^{s}(\mathbb{R}) \rightarrow \mathbb{R}, \quad(f, \varphi) \mapsto\langle f \circ \varphi, \psi\rangle
$$

is continuous for any testfunction $\psi$.

There is also the following chain rule

Lemma A.4. Let $s>3 / 2$ and $1 / 2<\tilde{s}<1$. For $\varphi \in \mathscr{D}^{s}(\mathbb{R})$ and $f \in H^{\tilde{s}}(\mathbb{R})$ we have

$$
\partial_{x}(f \circ \varphi)=f_{x} \circ \varphi \cdot \varphi_{x} .
$$

as an identity in $H^{\tilde{s}-1}(\mathbb{R})$.

Proof. Let $\psi$ be a testfunction. Then we have

$$
\begin{aligned}
\left\langle\partial_{x}(f \circ \varphi), \psi\right\rangle & =-\left\langle f \circ \varphi, \psi_{x}\right\rangle=-\left\langle f, \frac{\psi_{x} \circ \varphi^{-1}}{\varphi_{x} \circ \varphi^{-1}}\right\rangle=-\left\langle f, \partial_{x}\left(\psi \circ \varphi^{-1}\right)\right\rangle \\
& =\left\langle f_{x}, \psi \circ \varphi^{-1}\right\rangle=\left\langle f_{x} \circ \varphi, \psi \cdot \varphi_{x}\right\rangle=\left\langle f_{x} \circ \varphi \cdot \varphi_{x}, \psi\right\rangle
\end{aligned}
$$

which shows the claim.

We will also need the following Lipschitz type estimate.

Lemma A.5. Let $s>3 / 2$ and $\varphi_{\bullet} \in \mathscr{D}^{s}(\mathbb{R})$. There is a neighborhood $W \subseteq \mathscr{D}^{s}(\mathbb{R})$ of $\varphi_{\bullet}$ and a constant $C>0$ with

$$
\left\|f \circ \varphi_{1}-f \circ \varphi_{2}\right\|_{s-2} \leq C|| f\left\|_{s-1}\right\| \varphi_{1}-\varphi_{2} \|_{s-1}
$$

for all $\varphi_{1}, \varphi_{2} \in W$ and for all $f \in H^{s-1}(\mathbb{R})$.

Proof. Let $f \in C_{c}^{\infty}(\mathbb{R})$. We have from the fundamental lemma of calculus

$$
f\left(\varphi_{2}(x)\right)=f\left(\varphi_{1}(x)\right)+\int_{0}^{1} f^{\prime}\left(\varphi_{1}(x)+t\left(\varphi_{2}(x)-\varphi_{1}(x)\right)\right)\left(\varphi_{2}(x)-\varphi_{1}(x)\right) d t
$$

Taking $W$ small enough we can ensure that $\varphi_{1}+t\left(\varphi_{2}-\varphi_{1}\right) \in \mathscr{D}^{s}(\mathbb{R})$ for $0 \leq t \leq 1$. We thus see that

$$
t \rightarrow\left(f^{\prime} \circ\left(\varphi_{1}+t\left(\varphi_{2}-\varphi_{1}\right)\right)\right) \cdot\left(\varphi_{2}-\varphi_{1}\right)
$$

is continuous from $[0,1]$ to $H^{s}(\mathbb{R})$. As evaluation at $x \in \mathbb{R}$ is a continuous linear map on $H^{s}(\mathbb{R})$ we have the identity in $H^{s}(\mathbb{R})$

$$
f \circ \varphi_{2}-f \circ \varphi_{1}=\int_{0}^{1}\left(f^{\prime} \circ\left(\varphi_{1}+t\left(\varphi_{2}-\varphi_{1}\right)\right)\right) \cdot\left(\varphi_{2}-\varphi_{1}\right) d t
$$

where the integral is understood as a Riemann integral. We thus have

$$
\left\|f \circ \varphi_{2}-f \circ \varphi_{1}\right\|_{s-2} \leq \int_{0}^{1}\left\|f^{\prime} \circ\left(\varphi_{1}+t\left(\varphi_{2}-\varphi_{1}\right)\right)\right\|_{s-2}\left\|\varphi_{2}-\varphi_{1}\right\|_{s-1} d t
$$


For $W$ small enough we can ensure that

$$
\left\|f^{\prime} \circ\left(\varphi_{1}+t\left(\varphi_{2}-\varphi_{1}\right)\right)\right\|_{s-2} \leq C|| f^{\prime} \|_{s-2}
$$

Thus we get

$$
\left\|f \circ \varphi_{2}-f \circ \varphi_{1}\right\|_{s-2} \leq C|| f\left\|_{s-1}\right\| \varphi_{2}-\varphi_{1} \|_{s-1}
$$

For general $f \in H^{s-1}(\mathbb{R})$ we get the inequality by taking approximations $f_{n}$ because $f \mapsto f \circ \varphi$ is continuous in $H^{s-1}(\mathbb{R})$.

For the inversion map $\varphi \mapsto \varphi^{-1}$ we have

Lemma A.6. Let $s>3 / 2$ and $\varphi_{\bullet} \in \mathscr{D}^{s}(\mathbb{R})$. There is a neighborhood $W \subseteq \mathscr{D}^{s}(\mathbb{R})$ of $\varphi_{\bullet}$ and a constant $C>0$ with

$$
\left\|\varphi_{2}^{-1}-\varphi_{1}^{-1}\right\|_{s-1} \leq C|| \varphi_{2}-\varphi_{1} \|_{s}
$$

for all $\varphi_{1}, \varphi_{2} \in W$.

Proof. If $s>5 / 2$ then the lemma follows from [23] where it was shown that $\mathscr{D}^{s+1}(\mathbb{R}) \mapsto \mathscr{D}^{s}(\mathbb{R})$ is $C^{1}$. So it remains to check the case $s \leq 5 / 2$. Consider first the case $3 / 2<s<2$. We have

$$
\int_{\mathbb{R}}\left|\varphi^{-1}-\tilde{\varphi}^{-1}\right|^{2} d x=\int_{\mathbb{R}}\left|\varphi^{-1}-\varphi^{-1} \circ \varphi \circ \tilde{\varphi}^{-1}\right|^{2} d x
$$

As by the Sobolev imbedding the $C^{1}$-norm is bounded by the $H^{s}$-norm we have

$$
\left|\varphi^{-1}(x)-\varphi^{-1}(y)\right| \leq C|x-y|
$$

with a uniform $C$ in a neighborhood of $\varphi_{\bullet}$. Hence

$$
\int_{\mathbb{R}}\left|\varphi^{-1}-\tilde{\varphi}^{-1}\right|^{2} d x \leq C \int_{R} \mid x-\varphi\left(\left.\tilde{\varphi}^{-1}(x)\right|^{2} d x\right.
$$

By a change of variables we can bound the latter by

$$
C \int_{\mathbb{R}}|\tilde{\varphi}(x)-\varphi(x)|^{2} \tilde{\varphi}_{x}(x) d x
$$

As $\varphi_{x}$ is uniformly bounded in a neighborhood of $\varphi_{\bullet}$ we get

$$
\left\|\varphi^{-1}-\tilde{\varphi}^{-1}\right\|_{L^{2}} \leq C|| \varphi-\tilde{\varphi} \|_{s-1}
$$

Let us estimate the fractional part. Using the Sobolev-Slobodecki $\left[\varphi^{-1}-\tilde{\varphi}^{-1}\right]_{\lambda}$ norm with $\lambda=s-1$ we have

$$
\int_{\mathbb{R} \times \mathbb{R}} \frac{\left|\left[\varphi^{-1}(x)-\tilde{\varphi}^{-1}(x)\right]-\left[\varphi^{-1}(y)-\tilde{\varphi}^{-1}(y)\right]\right|^{2}}{|x-y|^{1+2 \lambda}} d x d y=\int_{\mathbb{R} \times \mathbb{R}} \frac{\left|\Phi\left(\tilde{\varphi}^{-1}(x)\right)-\Phi\left(\tilde{\varphi}^{-1}(y)\right)\right|^{2}}{|x-y|^{1+2 \lambda}} d x d y
$$

where

$$
\Phi(x)=\varphi^{-1}(\tilde{\varphi}(x))-\varphi^{-1}(\varphi(x))
$$


Change of variables gives

$$
\int_{\mathbb{R} \times \mathbb{R}} \frac{|\Phi(x)-\Phi(y)|^{2}}{|\tilde{\varphi}(x)-\tilde{\varphi}(y)|^{1+2 \lambda}} \tilde{\varphi}_{x}(x) \tilde{\varphi}_{x}(y) d x d y \leq C \int_{\mathbb{R} \times \mathbb{R}} \frac{|\Phi(x)-\Phi(y)|^{2}}{|x-y|^{1+2 \lambda}} \frac{|x-y|^{1+2 \lambda}}{|\tilde{\varphi}(x)-\tilde{\varphi}(y)|^{1+2 \lambda}} d x d y
$$

which by the fact that $K|\tilde{\varphi}(x)-\tilde{\varphi}(y)| \geq|x-y|$ holds is bounded by

$$
C \int_{\mathbb{R} \times \mathbb{R}} \frac{|\Phi(x)-\Phi(y)|^{2}}{|x-y|^{1+2 \lambda}} d x d y
$$

By the fundamental lemma of calculus

$$
\Phi(x)=\left(\int_{0}^{1} \frac{d t}{\varphi_{x} \circ \varphi^{-1}(\varphi(x)+t(\tilde{\varphi}(x)-\varphi(x)))}\right) \cdot(\tilde{\varphi}(x)-\varphi(x))=\Psi(x) \cdot(\tilde{\varphi}(x)-\varphi(x))
$$

Writing

$$
\Phi(x)-\Phi(y)=\Psi(x)([\tilde{\varphi}(x)-\varphi(x)]-[\tilde{\varphi}(y)-\varphi(y)])+(\Psi(x)-\Psi(y))(\tilde{\varphi}(y)-\varphi(y))
$$

Thus we can estimate

$$
[\Phi]_{\lambda} \leq \sup _{x \in \mathbb{R}}|\Psi(x)|[\tilde{\varphi}-\varphi]_{\lambda}+\sup _{x \in \mathbb{R}}|\tilde{\varphi}(x)-\varphi(x)|[\Psi]_{\lambda}
$$

Note that $[\Psi]_{\lambda}<\infty$ as

$$
t \mapsto \frac{1}{\varphi_{x} \circ \varphi^{-1}(\varphi(x)+t(\tilde{\varphi}(x)-\varphi(x)))}-1
$$

is a continuous path in $H^{s-1}(\mathbb{R})$. Therefore we get

$$
\left[\varphi^{-1}-\tilde{\varphi}^{-1}\right]_{\lambda} \leq C\|\varphi-\tilde{\varphi}\|_{s-1}
$$

Now consider the case $2 \leq s \leq 5 / 2$. Taking the derivative we get

$$
\left(\varphi^{-1}-\tilde{\varphi}^{-1}\right)^{\prime}=\varphi_{x} \circ \varphi^{-1}-\tilde{\varphi}_{x} \circ \tilde{\varphi}^{-1}
$$

which rewritten is

$$
\varphi_{x} \circ \varphi^{-1}-\varphi_{x} \circ \tilde{\varphi}^{-1}+\varphi_{x} \circ \tilde{\varphi}^{-1}-\tilde{\varphi}_{x} \circ \tilde{\varphi}^{-1}
$$

For the last two terms we have

$$
\left\|\varphi_{x} \circ \tilde{\varphi}^{-1}-\tilde{\varphi}_{x} \circ \tilde{\varphi}^{-1}\right\|_{s-2} \leq C|| \varphi_{x}-\tilde{\varphi}_{x}\left\|_{s-2} \leq C\right\| \varphi-\tilde{\varphi} \|_{s-1}
$$

For the first two terms we can argue as in the proof of Lemma A.5 and write

$$
\left\|\varphi_{x} \circ \varphi^{-1}-\varphi_{x} \circ \tilde{\varphi}^{-1}\right\|_{s-2} \leq C\left\|\varphi_{x}-1\right\|_{s-1}\left\|\varphi^{-1}-\tilde{\varphi}^{-1}\right\|_{s-2}
$$

Hence using the estimate from above for $\left\|\varphi^{-1}-\tilde{\varphi}^{-1}\right\|_{s-2}$ as $0 \leq s-2<1$ we get the claim. 


\section{Appendix B. Inequalities for fractional Sobolev functions}

In this section we will establish inequalities of the form

$$
\|f+g\| \geq C\left(\|f\|_{s}+\|g\|_{s}\right)
$$

for functions $f, g$ with disjoint support. For fractional $s$ this causes some difficulties as the norm $\|\cdot\|_{s}$ is defined in a non-local way. For fixed supports we have

Lemma B.1. Let $s \in \mathbb{R}$. There is a constant $C>0$ such that for all $f, g \in C_{c}^{\infty}(\mathbb{R})$ with $\operatorname{supp} f \subseteq$ $(-3,-1)$ and supp $g \subseteq(1,3)$ we have

$$
\|f+g\|_{s}^{2} \geq C\left(\|f\|_{s}^{2}+\|g\|_{s}^{2}\right)
$$

Proof. We take $\varphi, \psi \in C_{c}^{\infty}(\mathbb{R})$ with $\operatorname{supp} \varphi \subseteq(-3.5,-0.5)$ and $\operatorname{supp} \psi \subseteq(0.5,3.5)$ such that $\left.\varphi\right|_{(-3,-1)} \equiv 1$ and $\left.\psi\right|_{(1,3)} \equiv 1$. We then have

$$
\|f\|_{s}=\|\varphi(f+g)\|_{s} \leq C_{1}\|f+g\|_{s}
$$

and similarly

$$
\|g\|_{s}=\|\psi(f+g)\|_{s} \leq C_{2}\|f+g\|_{s}
$$

giving the desired result.

In the following we will use the fact that the $H^{s}$-norm is equivalent to the homogeneous $\dot{H}^{s}$ norm if we restrict ourselves to functions with support in a fixed compact $K \subseteq \mathbb{R}$ (see e.g. [4] p. 39). Recall

$$
\|f\|_{\dot{H}^{s}}^{2}=\int_{\mathbb{R}}|\xi|^{2 s}|\hat{f}(\xi)|^{2} d \xi
$$

We often also use $f^{\lambda}(x):=f(x / \lambda)$ for which we have the following scaling property

$$
\left\|f^{\lambda}\right\|_{\dot{H}^{s}}^{2}=\lambda^{1-2 s}\|f\|_{\dot{H}^{s}}^{2}
$$

We have

Lemma B.2. Let $s \geq 0$. Then there is a constant $C>0$ with the following property: For $x, y$ in $\mathbb{R}$ with $0<r:=|x-y| / 4<1$ we have

$$
\|f+g\|_{s}^{2} \geq C\left(\|f\|_{s}^{2}+\|g\|_{s}^{2}\right)
$$

for all functions $f, g \in C_{c}^{\infty}(\mathbb{R})$ with $\operatorname{supp} f \subseteq(x-r, x+r)$, supp $g \subseteq(y-r, y+r)$ 
Proof. We use the homogeneous norm. Now scaling with $\lambda=(4 r)^{-1}$ gives a situation as in Lemma B.1. We have

$$
\|f+g\|_{\dot{H}^{s}}^{2}=\lambda_{n}^{2 s-1}\left\|f^{\lambda}+g^{\lambda}\right\|_{\dot{H}^{s}}^{2}
$$

Now by Lemma B.1 we then get

$$
\|f+g\|_{\dot{H}^{s}}^{2} \geq C \lambda^{2 s-1}\left(\left\|f^{\lambda}\right\|_{\dot{H}^{s}}^{2}+\left\|g^{\lambda}\right\|_{\dot{H}^{s}}^{2}\right)
$$

Scaling back gives

$$
\|f+g\|_{\dot{H}^{s}}^{2} \geq C\left(\|f\|_{\dot{H}^{s}}^{2}+\|g\|_{\dot{H}^{s}}^{2}\right)
$$

This establishes the lemma.

We will encounter Lemma B.2 also for some negative values of $s$. In these cases we will use

Lemma B.3. Let $s<0$ and the same situation as in Lemma B.2. Then we have

$$
\|f+g\|_{s}^{2} \geq C\left(\|f\|_{s}^{2}+\|g\|_{s}^{2}\right)
$$

for all functions $f, g \in C_{c}^{\infty}(\mathbb{R})$ with $\operatorname{supp} f \subseteq(x-r, x+r)$, supp $g \subseteq(y-r, y+r)$

Proof. We claim that for functions with support in some fixed compact set $K \subseteq \mathbb{R}$ the homogeneous norm

$$
\|f\|_{\dot{H}^{s}}^{2}=\int_{\mathbb{R}}|\xi|^{2 s}|\hat{f}(\xi)|^{2} d \xi
$$

is equivalent to the non-homegeneous norm $\|\cdot\|_{s}$. One then can argues as in Lemma B.2 by scaling to a fixed situation as in Lemma B.1. So it remains to show the equivalence of the norms. We clearly have $\|\cdot\|_{s} \leq\|\cdot\|_{\dot{H}^{s}}$ since

$$
\int_{\mathbb{R}}\left(1+\xi^{2}\right)^{s}|\hat{f}(\xi)|^{2} d \xi \leq \int_{\mathbb{R}} \xi^{2 s}|\hat{f}(\xi)|^{2} d \xi
$$

For the other direction we use the dual definition of the Sobolev norm

$$
\|f\|_{s}=\sup _{\|g\|_{-s} \leq 1}|\langle f, g\rangle|
$$

and analogously for the homogeneous norm. Taking $\psi \in C_{c}^{\infty}(\mathbb{R})$ with $\psi=1$ on $K$ we have for $f$ with support in $K$

$$
\|f\|_{\dot{H}^{s}}=\sup _{\|g\|_{\dot{H}^{-s}} \leq 1}|\langle f, \psi \cdot g\rangle|
$$

Now note that we have equivalence of the norms $\|\cdot\|_{-s}$ and $\|\cdot\|_{\dot{H}^{-s}}$ for functions with support in some fixed compact. Therefore

$$
\begin{aligned}
\|f\|_{\dot{H}^{s}} & =\sup _{\|g\|_{\dot{H}^{s}} \leq 1}|\langle f, \psi \cdot g\rangle| \leq \sup _{g,\|\psi \cdot g\|_{-s} \leq C_{1}}|\langle f, \psi \cdot g\rangle| \\
& \leq C_{1} \sup _{\|g\|_{-s} \leq 1}|\langle f, g\rangle|=\|f\|_{-s}
\end{aligned}
$$

showing the equivalence. 


\section{References}

[1] R. Abraham and J. Robbin, Transversal mappings and flows, W.A. Benjamin, Inc., New York, 1967.

[2] R. Adams, Sobolev spaces, Academic Press, New York, 1975.

[3] V. Arnold: Sur la géometrie differentielle des groupes de Lie de dimension infinie et ses applications à l'hydrodynamique des fluids parfaits, Ann. Inst. Fourier, 16, 1(1966), 319-361.

[4] H. Bahouri, J-Y. Chemin, R. Danchin: Fourier analysis and nonlinear partial differential equations, Grundlehren der Mathematischen Wissenschaften [Fundamental Principles of Mathematical Sciences], 343. Springer, Heidelberg, 2011

[5] R. Camassa, D. Holm: An integrable shallow water equation with peaked solitons, Phys. Rev. Lett, 71(1993), 1661-1664.

[6] M. Cantor: Groups of diffeomorphisms of $R^{n}$ and the flow of a perfect fluid, Bull. Am. Math. Soc. 81, 205-208 (1975)

[7] A. Constantin:Existence of permanent and breaking waves for a shallow water equation: a geometric approach, Ann. Inst. Fourier 50, No.2, 321-362 (2000)

[8] A. Constantin, J. Escher: Well-posedness, global existence, and blow-up phenomena for a periodic quasi-linear hyperbolic equation, Commun. Pure Appl. Math., 51(1998), 475-504.

[9] A. Constantin, D. Lannes: The hydrodynamical relevance of the Camassa-Holm and DegasperisProcesi equations, Arch. Ration. Mech. Anal. 192 (2009), no. 1, 165186.

[10] A. Constantin, H. P. McKean: A shallow water equation on the circle., Comm. Pure Appl. Math. 52 (1999), no. 8, 949982.

[11] A. Constantin, R. I. Ivanov, J. Lenells: Inverse scattering transform for the Degasperis-Procesi equation., Nonlinearity 23 (2010), no. 10, 25592575.

[12] A. Constantin, T. Kappeler, B. Kolev, P. Topalov: On geodesic exponential maps of the Virasoro group, Ann. Glob. Anal. Geom. 31(2007), 155 - 180.

[13] O. Christov, S. Hakkaev: On the Cauchy problem for the periodic b-family of equations and of the nonuniform continuity of Degasperis-Procesi equation, J. Math. Anal. Appl. 360, No. 1, 47-56 (2009).

[14] A. Degasperis, M. Procesi: Asymptotic integrability, in Symmetry and Perturbation Theory, Rome, World Sci. Publ., River Edge, NJ, 1999

[15] D. Ebin, J. Marsden: Groups of diffeomorphisms and the motion of an incompressible fluid, Ann. Math., 92(1970), 102-163.

[16] J. Escher, D. Henry, B. Kolev, T. Lyons: Two-component equations modelling water waves with constant vorticity, Annali Mat. Pura Appl. (http://link.springer.com/article/10.1007/s10231-014-0461-z)

[17] J. Escher, B. Kolev: The Degasperis-Procesi equation as a non-metric Euler equation, Math. Z. 269 (2011), no. 3-4, 11371153.

[18] J. Escher, B. Kolev: Geometrical methods for equations of hydrodynamical type., J. Nonlinear Math. Phys. 19 (2012), suppl. 1

[19] J. Escher, T. Lyons: Two-component higher order Camassa-Holm systems with fractional inertia operator: a geometric approach., J. Geom. Mech. 7 (2015), no. 3, 281293.

[20] A. Himonas, C. Kenig, G. Misiolek: Non-uniform dependence for the periodic CH equation, Commun. Partial Differ. Equations 35, No. 6, 1145-1162 (2010)

[21] A. Himonas, G. Misiolek: The Cauchy problem for an integrable shallow-water equation., Differ. Integral Equ. 14, No.7, 821-831 (2001)

[22] D. Holm, M. Staley:Nonlinear balance and exchange of stability in dynamics of solitons, peakons, ramps/cliffs and leftons in a $1+1$ nonlinear evolutionary PDE, Phys. Lett., A 308, No.5-6, 437-444 (2003).

[23] H. Inci, T. Kappeler, P. Topalov: On the regularity of the composition of diffeomorphisms, Mem. Amer. Math. Soc. 226 (2013), no. 1062

[24] H. Inci: On the well-posedness of the incompressible Euler Equation, thesis, arXiv:1301.5997 (2013)

[25] H. Inci: On a Lagrangian formulation of the incompressible Euler equation, arXiv:1301.5994 (2013)

[26] H. Inci: On the regularity of the solution map of the incompressible Euler equation, Dyn. Partial Differ. Equ. 12 (2015), no. 2, 97113

[27] S. Lang: Fundamentals of Differential Geometry, Springer, 1999. 
[28] G.Misiolek:A shallow water equation as a geodesic flow on the Bott-Virasoro group, J. Geom. Phys. 24, No.3, 203-208 (1998).

[29] Y. Li, P. Olver: Well-posedness and blow-up solutions for an integrable nonlinearly dispersive model wave equation, J. Differential Equations 162 (2000), 27-63.

[30] V.Yu. Ovsienko, B.A. Khesin:The Korteweg-de Vries superequation as Euler equation, Funkts. Anal. Prilozh. 21, No.4, 81-82 (1987).

[31] G. Rodriguez-Blanco: On the Cauchy problem for the Camassa-Holm equation, Nonlinear Anal. 14 (2001), 309-327.

[32] L. Yan: Nonuniform dependence for the Cauchy problem of the general b-equation, J. Math. Phys. 52, 033101 (2011) 